\title{
A RLS Filter for Nonuniformity and Ghosting Correction of Infrared Image Sequences ${ }^{\star}$
}

\author{
Flavio Torres ${ }^{1}$, César San Martin ${ }^{1,2}$, and Sergio N. Torres ${ }^{2}$ \\ ${ }^{1}$ Department of Electrical Engineering, University of La Frontera, \\ Casilla 54-D, Temuco, Chile \\ ftorres@ufro.cl \\ ${ }^{2}$ Department of Electrical Engineering, University of Concepción, \\ Casilla 160-C, Concepción, Chile \\ sertorre@udec.cl \\ http://nuc.die.udec.cl
}

\begin{abstract}
In this paper, a technique to improve the convergence and to reduce the ghosting artifacts of a previously developed adaptive scenebased nonuniformity correction method is presented. The nonuniformity correction method estimates detector parameters based on the recursive least square filter approach. We propose, three parameters to reduce ghosting artifacts and to speed up the convergence of such method by using only the read-out data. The parameters proposed are based in identify global motion between consecutive frames as well as evaluate the main assumption used in the previous method in the uncertainty on the input infrared irradiance. The ability of the method to compensate for nonuniformity and reducing ghosting artifacts is demonstrated by employing several infrared video sequences obtained using two infrared cameras.
\end{abstract}

Keywords: Image Sequence Processing, Infrared Imaging, RLS.

\section{Introduction}

Infrared imaging systems are employed in several applications such as defense, astronomy and medical science. In general, those systems are based on the infrared focal-plane array IR FPA technology. An IR-FPA is a die composed of a group of photodetectors placed in a focal plane forming a matrix of $X \times Y$ pixels, which gives the sensor the ability to collect the IR information.

It is well known that nonuniformity noise in IR imaging sensors, which is due to pixel-to-pixel variation in the detectors' responses, can considerably degrade the quality of IR images since it results in a fixed-pattern-noise (FPN) that is superimposed on the true image. Even more, what makes matter worse is that the

\footnotetext{
* This work was supported by Universidad de La Frontera, Proyecto DIUFRO EP N ${ }^{o}$ 06-0253 . The authors wish to thank Ernest E. Armstrong (OptiMetrics Inc., USA) and Pierre Potet (CEDIP Infrared Systems, France) for collecting the data.
} 
nonuniformity slowly varies over time, and depending on the technology used, this drift can take from minutes to hours. In order to solve this problem, several scene-based nonuniformity correction (NUC) techniques have been developed 1 12 2 34456 . Scene-based techniques perform the NUC using only the video sequences that are being imaged, not requiring any kind of laboratory calibration technique.

Our group has been given special attention to NUC methods based on estimation theory. Seeking for more effectiveness in the reduction of NUC, we propose to improve our previously published adaptive scene-based NUC method [7], based in a RLS (recursive least square) filter [8]. The NUC method based in a RLS algorithm exhibits the advantages of fast convergence rate and unbiased stationary error 910. However, the method has shown the following weakness: the supposition that the input scene is constantly moving, in general, is not valid and ghosting artifacts are generated; the assumption that the best target for the unknown input infrared irradiance is an average over its neighboring pixels is scene dependent generating a poor correction in pixels, which are part of objects boundaries. Then, for improving the performance of the NUC RLS algorithm, we propose three parameters to mitigate the ghosting artifacts and speed up the convergence in pixels that are part of the edges of objets being imaged. The effect of the parameters are tested with simulated and real IR data.

This paper is organized as follows. In Section 2 the IR-FPA model and the RLS- NUC method with the proposed enhancement are presented. In Section 3 the RLS- NUC technique is tested with video sequences of real raw IR data captured using two infrared cameras. In Section 4 the conclusions of the paper are summarized.

\section{The RLS NUC Method for Infrared Video Sequences}

In this section, the previously published scene-based NUC method [7] is presented for completeness. We begin reviewing the most common model used for the nonuniformity presented IR-FPA technology, and we finish developing three parameters with the ability of being used to reduce ghosting artifacts and to speed up convergence of such method.

\subsection{RLS Filter Method}

In this paper, we adopt the commonly used linear model for the infrared detector. For the $(i j)^{\text {th }}$ detector in IR-FPA, the measured read-out signal $Y_{i j}$ at a given time $n$ can be expressed as:

$$
Y_{i j}(n)=g_{i j}(n) \cdot X_{i j}(n)+o_{i j}(n)+v_{i j}(n)
$$

where $g_{i j}(n)$ and $o_{i j}(n)$ are the gain and the offset of the $i j^{\text {th }}$ detector, $X_{i j}(n)$ is the real incident IR photon flux collected by the respective detector, and $v_{i j}(n)$ is the additive electronic noise. Re-writing equation (1) in a vectorial form we obtain:

$$
Y_{i j}(n)=\Psi_{i j}^{T}(n) \Theta_{i j}(n)+v_{i j}(n)
$$


where, $\Psi_{i j}(n)=\left[X_{i j}(n), 1\right]^{T}$ is the IR data vector and $\Theta_{i j}(n)=\left[g_{i j}(n), o_{i j}(n)\right]^{T}$, is the detector parameter vector. Then, an estimator for $\Theta_{i j}$ can be formulated like:

$$
\hat{Y}_{i j}(n)=\Psi_{i j}^{T}(n) \hat{\Theta}_{i j}(n)
$$

and the estimation error is given by:

$$
e_{i j}(n)=Y_{i j}(n)-\Psi_{i j}^{T}(n) \hat{\Theta}_{i j}(n)
$$

From (3) is possible to obtain a solution only if the unknown real incident IR is known. The key assumption of the method is that $X_{i j}$ can be initially estimated from the read-out data $Y_{i j}$. Then, we initially estimate the real IR $X_{i j}$ with the spatial mean value of the corrupted image at $i j$ sensor [4], that is:

$$
X_{i j}(n)=\bar{Y}_{i j}(n)=\frac{1}{(2 v+1)^{2}} \sum_{k=i-v}^{i+v} \sum_{l=j-v}^{j+v} Y_{k l}(n)
$$

where $\bar{Y}$ is a smoothing version of $Y$ and $v$ fixes the averaging window size. This assumption is valid when the scene is constantly moving with respect to the detector. Because $\bar{Y}$ is more uniform than $Y$, a spatial nonuniformity correction is performed. Then, equation (3) will be used to estimate the detector parameters with $\Psi_{i j}(n)=\left[\bar{Y}_{i j}(n), 1\right]^{T}$, i.e., we suppose that the gain parameters have a spatial normal distribution with unit mean, and the offset have a spatial normal distribution with zero mean.

Then, we minimize the functional

$$
\epsilon_{i j}(n)=\sum_{k=1}^{n} \lambda_{i j}^{n-k} e_{i j}^{2}(n)
$$

where $\lambda$ is the forgetting factor which varies to in $0<\lambda<1$, and weighs the influence of past error values. Deriving $\epsilon_{i j}(n)$ with respect to the parameter vector $\Theta_{i j}(n)$, equalling to zero and solving for the parameter vector, we obtain

$$
\hat{\Theta}_{i j}(n)=\left[\sum_{k=1}^{n} \lambda^{n-k} \Psi_{i j}(k) \Psi_{\mathrm{i} j}^{T}(k)\right]^{-1} \sum_{k=1}^{n} \lambda^{n-k} \Psi_{i j}(k) y_{i j}(k)=P_{i j}(n) \psi_{i j}(n)
$$

For a recursive update of the parameters, the RLS algorithm is used, hence all necessary equations to form the algorithm are:

$$
\begin{gathered}
\hat{\Theta}_{i j}(n+1)=\hat{\Theta}_{i j}(n)+\mathrm{K}_{i j}(n+1)\left[Y_{i j}(n+1)-\Psi_{i j}^{T}(n+1) \hat{\Theta}_{i j}(n)\right] \\
\mathrm{K}_{i j}(n+1)=\mathrm{P}_{i j}(n) \Psi_{i j}(n+1)\left[\lambda-\Psi_{i j}^{T}(n+1) \mathrm{P}_{i j}(n) \Psi_{i j}(n+1)\right]^{-1} \\
\mathrm{P}_{i j}(n+1)=\left[I-\mathrm{K}_{i j}(n+1) \Psi_{i j}^{T}(n+1)\right] \mathrm{P}_{i j}(n) \cdot \frac{1}{\lambda}
\end{gathered}
$$


where, $\hat{\Theta}_{i j}(n)=\left[\hat{g}_{i j}(n), \hat{o}_{i j}(n)\right]^{T}$, is the estimated parameter vector, $\mathrm{K}_{i j}(n)$ is the gain vector and $\mathrm{P}_{i j}(n)$ is the covariance matrix.

Finally, to remove the nonuniformity noise of the corrupted image sequence, we use the parameters estimated by the RLS algorithm like the following:

$$
\hat{X}_{i j}(n)=Y_{i j}(n) / \hat{g}_{i j}(n)-\hat{o}_{i j}(n) / \hat{g}_{i j}(n)
$$

where $\hat{X}_{i j}(n)$ is the corrected image.

\subsection{Performance of the RLS NUC Method}

The main problems detected on the performance of the RLS NUC method are originated by three basic constraints used in the development of the method. The first is related to the supposition that the scene is constantly moving with respect to the detector. Then, the problem is that when the scene is not moving for a few frames, this equality is not valid, and the ghosting effect appears. This consists in a ghost present in the next frames.

The second is related to the supposition that the readout data has imaged object with low spatial diversity in the integrate IR radiation, which in the general case, is also not valid. The spatial uniformity in the image is used to obtain an accurate parameter estimation of the RLS NUC method because the spatial variance of the read-out data and the true IR data are near to zero, and only variance of the offset and the electronic noise are presented.

The third constraint is that the RLS NUC method does not satisfies the orthogonality principle. This means that the variance of the error is not minimal. This generates an estimation with a considerable variability in the error.

In the next section, we propose parameters to be included in the algorithm to reduce the effects generated by the foregoing constraints.

\subsection{Nonuniformity and Ghosting Correction Algorithm}

The algorithm is improved considering three parameters, which are based on the following: motion detection, nonuniformity degree estimation and error variance. We propose an indicator for the motion detection between consecutive frames, i.e., between frames at $n$ and $n-1$ as follows:

$$
z_{1}(n)=\sum_{k, l}\left|Y_{n}(k, l)-Y_{n-1}(k, l)\right|
$$

where $z_{1}(n)$ is used to reduce ghosting. If $z_{1}(n)$ is close to zero, ghosting is presented on the corrected image. And, we propose an indicator for spatial variance of the IR radiation by considering the local spatial variance of $Y_{i j}(n), \sigma_{Y_{i j}(n)}^{2}$ calculated by

$$
\sigma_{Y_{i j}(n)}^{2}=\frac{1}{(2 v+1)^{2}} \sum_{k=i-v}^{i+v} \sum_{l=j-v}^{j+v}\left(Y_{k l}(n)-\bar{Y}_{i j}(n)\right)^{2}
$$


The frame by frame calculus allows us to estimate a new temporal variable $z_{2}(n)=\sigma_{Y_{i j}(n)}^{2}$ that is used as an indicator in the algorithm. Note that, small values of $z_{2}(n)$ means that the input data meets the required assumption, on the IR radiance, by the proposed method.

The last indicator is obtained using the error defined in equation (44) as $z_{3}(n)=$ $e_{i j}^{2}(n)$, i.e., the error variance. In this case, a good performance of the method takes place when $z_{3}(n)$ is minimal.

Then, the NUC algorithm is enhanced by the use of these parameters as described in the following steps:

- For each frame, we obtain $z_{1}(n), z_{2}(n)$ and $z_{3}(n)$,

- After $L$ frames, $\sigma_{z_{2}}^{2}$ is calculated

- Then, we calculate the $H$ value, defined as

$$
H=\frac{\sigma_{z_{2}}^{2} z_{3}}{1+z_{1}}
$$

- If at the current iteration, the calculated value of $\mathrm{H}$ is lower in magnitude than any previously calculated value, the estimated gain and offset are updated on this iteration. Otherwise, the gain and offset calculated at the previous iteration are not updated.
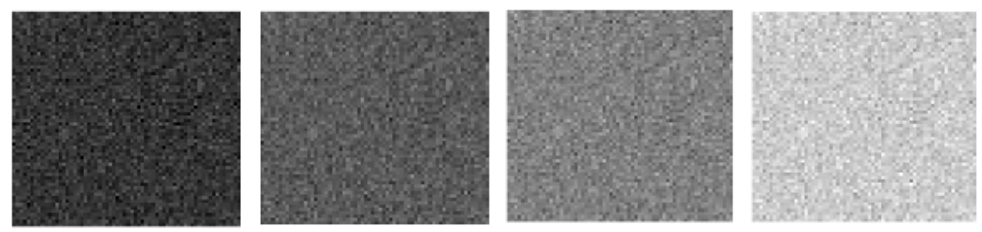

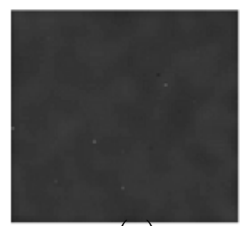

(a)

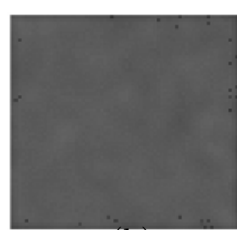

(b)

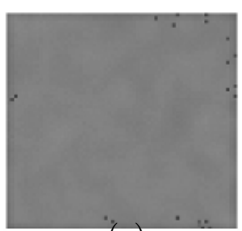

(c)

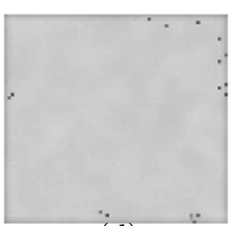

(d)

Fig. 1. Four frames of a simulated black bodies sequence (first row) and the correction with the enhanced NUC RLS method (second row). a) The 335 - th frame. b) The 500 - th frame. c) The 665 - th frame. d) The 845 - th frame.

\section{Evaluation of the Proposed Methods Upgrades}

The main goal of this section is to test the ability of the proposed method to reduce nonuniformity on simulated and real infrared video data. In the first place, we use simulated black body images. In the second place, the algorithm is tested with two real infrared image sequences taken by two different IR cameras. 
As a quantitative measure of performance, we use the Root Mean Square Error (RMSE), which measures the difference between the true infrared image with the corrected image using the proposed method. The RMSE is calculated by:

$$
R M S E(n)=\left\{\frac{1}{p m} \sum_{i=1}^{p} \sum_{j=1}^{m}\left(\hat{x}_{i j}(n)-x_{i j}(n)\right)^{2}\right\}^{1 / 2}
$$
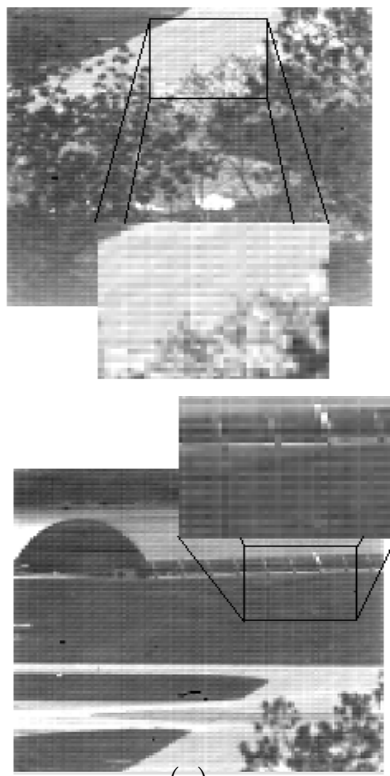

(a)
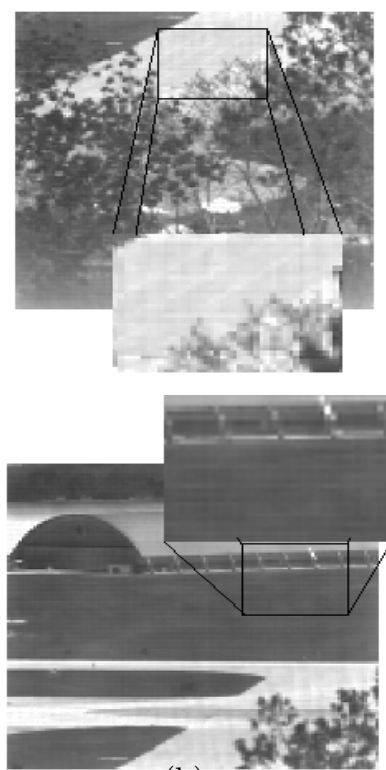

(b)
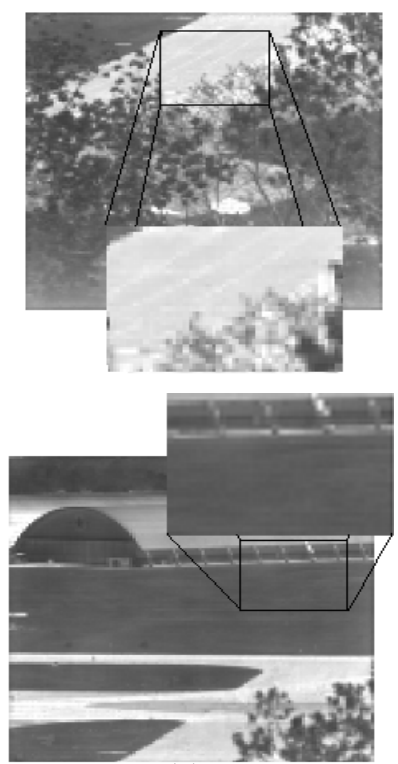

(c)

Fig. 2. Results on Real IR data with emphasis in ghost artifacts reducction. The first row shows the 770- th frame and the second row shows the 1600-th frame of the first set of IR data, a) The raw corrupted frames, b) The corresponding frames corrected by the previous RLS method [7] and c) The corresponding frames corrected by the enhanced method.

where $p \times m$ is the number of detectors in the FPA. A low value of RMSE means a better correction of the frame data. The evaluation procedure for each set of data is detailed in the following sections.

\subsection{Results with Simulated Black Bodies}

The simulated black bodies has been generated using flat images, where the intensity or gray level indicates the black bodies temperature. FPN is added to each image generating the corrupted sequence. An example is presented in the first row of Fig. 1. Those images present only nonuniform noise, hence the black 
bodies constitute a good test for the NUC algorithm. In the second row of Fig. [1 it is clear, using the naked eye, that the proposed method reduced the FPN and performed an accurate nonuniformity correction.

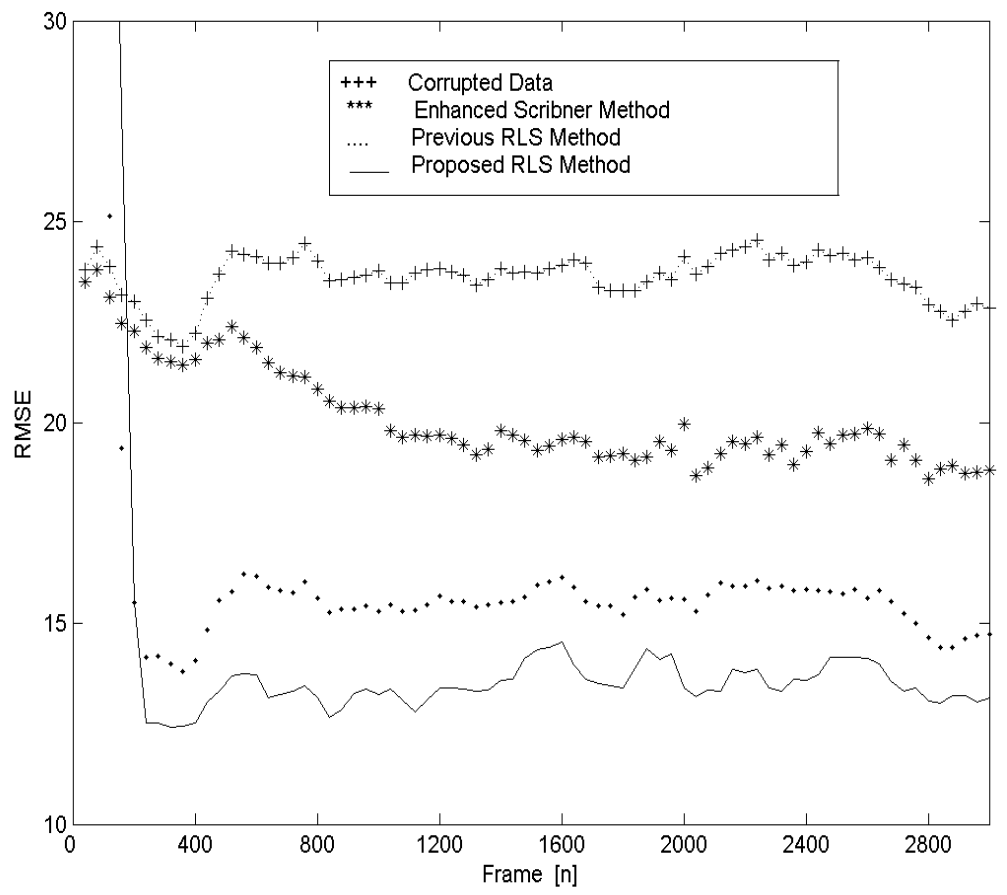

Fig. 3. The evolution of the RMSE between the reference (set 1 calibrated with black bodies) and the corrected frames of IR data set 1 . Line with a plus represents the corrupted data; line with a star represents the RMSE computed for the enhanced Scribners NUC method 4; line with a dot represents the previous NUC RLS method 7]; and solid line represents the RMSE computed for the proposed enhanced NUC-RLS method.

\subsection{Results with Two Real Infrared Image Sequences}

The first sequence has been collected using a $128 \times 128 \mathrm{InSb}$ FPA cooled camera (Amber Model AE-4128) operating in the $3-5 \mu m$ range. As an example, Fig. $2 \mathrm{a}$ shows a corrupted readout data frame. In Fig. 2 $2 \mathrm{~b}$ and $2 \mathrm{c}$ the corresponding corrected frame by the NUC RLS method and the enhance RLS method are presented, respectively. In Fig. 20 and Fig. 2ro we have shown a zooming of the corrected image, specifically of the zones indicated by boxes. In them, using only the naked eye is clear that the non-uniformity is notably better in the proposed NUC RLS method (Fig. 2 2 ) than in the previous published method (Fig. 2b).

The NUC performance, in this case, is evaluated by employing the index RMSE computed between a reference (the real IR sequence calibrated with black 
bodies) and the corrected IR video sequence. Figure 3 shows the calculated RMSE for the corrupted data, for each frame corrected using enhanced Scribner's NUC method [4, the RLS, and the enhanced RLS method. Further, the average RMSEs computed for the whole infrared sequence are equal to 20.15, 16.62 and 14.23 for the Scribner NUC method, the NUC-RLS algorithm and the enhanced NUC RLS algorithm, respectively. It can be seen in Fig. 3 that the RMSE value obtained for the enhanced NUC RLS method has a greater convergence speed, because, for the same number of frame, it reaches a lower RMSE value than any other method.
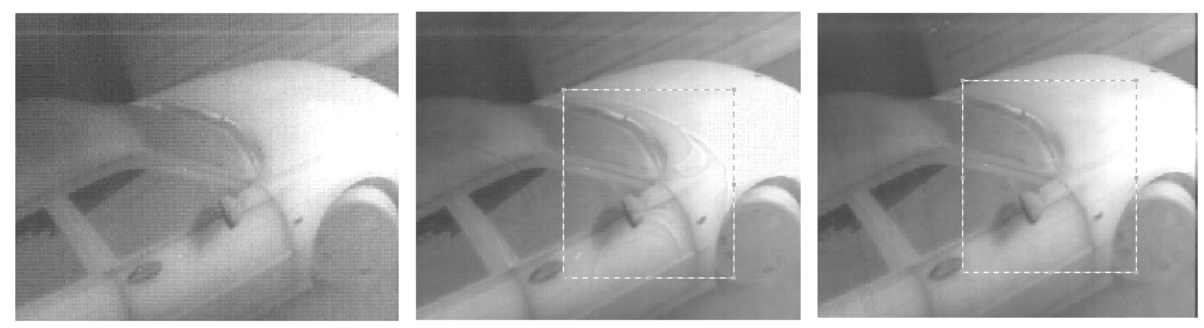

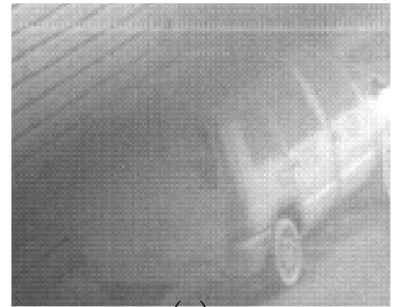

(a)

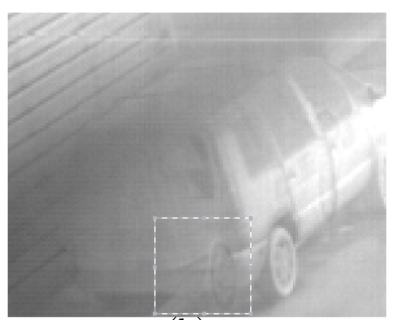

(b)

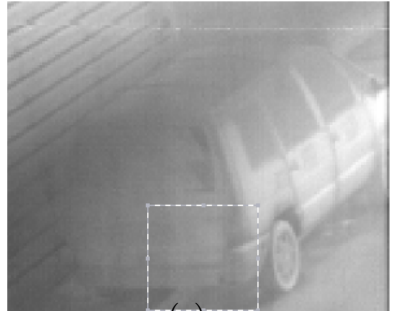

(c)

Fig. 4. Performance of the enhanced NUC-RLS method under real IR data. The first row shows the 297- th frame and the second row shows the 467-th frame of the second set of IR data, a) The raw corrupted frames, b) The corresponding frames corrected first by the RLS method and c) The corresponding frames corrected by the proposed enhance method.

The second sequence of infrared data has been recorded using a $320 \times 240$ $\mathrm{HgCdTe}$ FPA cooled camera (CEDIP Jade Model) operating in the $8-12 \mu \mathrm{m}$ range. As an example, Fig. 近 shows the corrupted readout data for two frames. In Fig. 4 b and Fig. 4k the corresponding corrected frames by the previous published method and the NUC method proposed in this paper are shown, respectively. The non-uniformity, visually presented in the raw frame, has been notably reduced by the proposed method; in Fig. 固 and Fig. 迎 this is specially noticed in the marked region. In Fig. 4 $4 \mathrm{~b}$ it is possible to appreciate the ghosting present on both frames. This ghost effect disappears when we used the RLS method with nonuniformity and ghosting correction algorithm, as it is shown in Fig. 4r. Thus, we have shown experimentally with real IR data that the proposed scenebased NUC RLS method with ghost correction has the ability of notably reduce 
both the non-uniformity noise presented in IR-FPA sensors and the ghosting generated by lack of motion in the recorded IR data.

\section{Conclusions}

In this paper an enhanced version of our previously published NUC method, based in a RLS filter is presented. The new method has the ability to improve the nonuniformity correction and to eliminate ghosting artifacts more efficiently than the previous method. This is obtained by means of three parameters based in identifying global motion between consecutive frames as well as, evaluate the main assumption used in the previous method in the uncertainty on the input infrared irradiance. Furthermore, the evaluation with simulated and real data has demonstrated that the proposed method reduces ghosting artifacts and improves the RMSE parameter when compared with the previous published method. Using read-out data taken from cameras of two different technologies we were able to observe that the method is capable to reduce nonuniformity, minimizing ghosting, with fast convergence and low RMSE.

\section{References}

1. Torres, S., Hayat, M.: Kalman Filtering for Adaptive Nonuniformity Correction in Infrared Focal Plane Arrays. The JOSA-A Opt. Soc. of America. 20. (2003) 470-480.

2. Torres, S., Pezoa, J., Hayat, M.: Scene-based Nonuniformity Correction for Focal Plane Arrays Using the Method of the Inverse Covariance Form. OSA App. Opt. Inf. Proc. 42. (2003) 5872-5881.

3. Scribner, D., Sarkady, K., Kruer, M.: Adaptive Nonuniformity Correction for Infrared Focal Plane Arrays using Neural Networks. Proceeding of SPIE. 1541. (1991) 100-109.

4. Scribner, D., Sarkady, K., Kruer, M.: Adaptive Retina-like Preprocessing for Imaging Detector Arrays. Proceeding of the IEEE International Conference on Neural Networks. 3. (1993) 1955-1960.

5. Torres, S., Vera, E., Reeves, R., Sobarzo, S.: Adaptive Scene-Based Nonuniformity Correction Method for Infrared Focal Plane Arrays. Proceeding of SPIE. 5076. (2003) 130-139.

6. Vera, E., Torres, S.: Fast Adaptive Nonuniformity Correction for Infrared Focal Plane Arrays. EURASIP Journal on Applied Signal Processing. (2005).

7. Torres, F., Torres, S., San Martin, C.: A Recursive Least Square Adaptive Filter for Nonuniformity Correction of Infrared Image Sequences. LNCS, 3773. (2005) $540-546$.

8. L. Ljung and T. Sderstrm: Theory and practice of recursive identification, MIT Press, Cambridge, 1983.

9. E. Eleftheriou, D.D. Falconer, Tracking properties and steady-state performance of RLS adaptive filter algorithms, IEEE Trans. Acoust. Speech Signal Process. ASSP 34 (1986) 1097-1110.

10. E. Ewada, Comparasion of RLS, LMS and sign algorithms for tracking randomly time-varying channels, IEEE Trans. Signal Process. 42 (1994) 2937-2944. 